J. Lake Sci.(湖泊科学), 2009, 21(4): 483-489

http://www.jlakes.org. E-mail: jlakes@niglas.ac.cn

(C2009 by Journal of Lake Sciences

\title{
五种天然水体胶体相可酶解磷的含量及分布特征
}

\author{
王 芳 ${ }^{1,3}$, 朱广伟 $1^{* *}$, 贺再再 ${ }^{2}$ \\ (1: 中国科学院南京地理与湖泊研究所湖泊与环境国家重点实验室, 南京 210008) \\ (2: 南京农业大学资源与环境科学学院环境工程系, 南京 210095) \\ (3: 中国科学院研究生院, 北京 100049)
}

摘 要: 分别在太湖草型湖区胥口湾及藻型湖区梅梁湾采集水样, 采用常规过滤与切向流超滤相结合的方法, 将水体中的颗 粒/胶体依据粒径大小分离, 通过测定计算得到 64-1 $\mu \mathrm{m}, 1-0.5 \mu \mathrm{m}, 0.5-0.1 \mu \mathrm{m}, 0.1 \mu \mathrm{m}-1 \mathrm{~nm},<1 \mathrm{~nm}$ 五个粒级的可酶解磷含量. 同 时采集太湖“引江济太”工程长江调水口——望虞河口水样、千岛湖水样及千岛湖下游河流型水库富春江段水样进行分析. 结 果显示, 5 种典型水体中颗粒相的可酶解磷含量占总可酶解磷含量的比重较高, 胶体相的可酶解磷也占了相当的比重, 是生物 必需磷不可忽视的重要储库. 望虞河口河水总磷 $0.216 \mathrm{mg} / \mathrm{L}$, 可酶解磷含量达 $0.174 \mathrm{mg} / \mathrm{L}$, 其输人可能对太湖水体生物有效磷 浓度的增加起到重要的贡献, “引江济太”调水时具有一定生态风险. 对于胶体范围的可酶解磷, 胥口湾、望虞河的胶体态可酶 解磷主要分布在较大、中胶体范围, 梅梁湾和千岛湖的胶体态可酶解磷主要分布在中、微胶体范围, 富春江水库的胶体态可 酶解磷分布的相对比较均匀.

关键词: 太湖; 胶体; 可酶解磷; 切向流超滤; 富营养化; 千岛湖; 富春江

\section{Distribution of colloidal enzymatically hydrolysable phosphorus in natural waters}

WANG Fang ${ }^{1,3}$, ZHU Guangwei ${ }^{1} \&$ HE Ranran ${ }^{2}$

(1: State Key Laboratory of Lake Science and Environment, Nanjing Institute of Geography and Limnology, Chinese Academy of Sciences, Nanjing 210008, P.R.China)

(2: College of Resource and Environmental Science, Department of Environmental Engineering, Nanjing Agricultural University, Nanjing 210095, P.R.China)

(3: Graduate University of Chinese Academy of Sciences, Beijing 100049, P.R.China)

Abstract: Contents of enzymatically hydrolysable phosphorus (EHP) in particle and colloidal phases in Lake Taihu were analyzed. Colloid in lake water was separated by tangential flow ultrafiltration, which included the component with diameters from $1 \mathrm{~nm}$ to 1000nm. The EHPs from Wangyu River, a large water channel connected Lake Taihu with Yangtze River, and from Lake Qiandao and Fuchun River were also studied. Mean of particle EHP (particle size larger than 1000nm) accounted 58\% of total EHP, which was the main contributor in the water. Mean of colloidal EHP accounted 35\% of total, which also shows a high ratio compared to the true dissolved phosphorus. During the investigation period, total phosphorus and EHP in Wangyu River were $0.216 \mathrm{mg} / \mathrm{L}$ and $0.174 \mathrm{mg} / \mathrm{L}$, respectively, suggesting that there is a high ecological risk to pump too much Yangtze River water to Lake Taihu. In Lake Taihu, percentage of micro-size colloid in marcophyte dominated zone (Xukou Bay) was much higher than that in cyanobacteria dominated zone (Meiliang Bay).

Keywords: Enzymatically hydrolysable phosphorus; colloid; eutrophication; Lake Taihu; ultrafiltration; Lake Qiandao; Fuchun River

长江中下游地区独特的地貌特征，加上近年来人类活动导致的流域营养物质输人量的过度增加，使

* 国家自然科学基金项目(40673078, 40730529)资助. 2008-10-07 收稿; 2009-03-16 收修改稿. 王芳, 女, 1984 年生, 硕士; E-mail:wfssn2002@163.com. 
得该地区湖泊普遍面临着富营养化问题，而蓝藻水华的暴发是水体富营养化的重要表征 ${ }^{[1]}$. 磷是许多湖 泊中浮游植物生长及水华形成的限制性营养盐 ${ }^{[2-3]}$, 因而磷在水体和沉积物中的含量、形态及迁移转化一 直以来受到广泛的关注 ${ }^{[4-6]}$. 而在富营养化湖泊治理过程中, 即使切断了外源负荷, 在较长的时期内水体 的磷仍然不能得到有效的降低, 这使得大量的研究集中到了湖泊的内源磷释放方面 ${ }^{[7-8]}$.

天然湖泊水体中总磷含量只能反映出很有限的信息，磷的生物可利用性、环境行为及生态效应 更多受到磷的物理和化学形态影响。水体中溶解性磷和悬浮态颗粒磷占到总磷的 $12 \%-30 \%$ 和 $62 \%-82 \%$, 生物可直接利用的溶解性反应磷 (DRP)仅占 $5 \%-8 \%$ 㟶, 然而, 藻类繁殖过程中及其他水 体相关代谢过程中, 生物体能够通过碱性磷酸酶等物质的催化作用, 促进各种非生物直接利用态的 磷转化为生物利用态的磷 ${ }^{[10-11]}$. 研究表明, 海域中尤其是河口和近岸海域颗粒态潜在的生物可利用 态磷在生物可利用磷中所占比例较高, 是生物可利用磷的一个不可忽视的来源和重要储库, 也是控 制海域磷供应水平的重要因子 ${ }^{[12-16]}$. 张敏等也发现, 水柱中的颗粒态磷与微囊藻的生物量呈显著的 正相关关系 ${ }^{[8]}$. 因此, 探讨水体中不同形态磷的可酶解性对认识湖泊蓝藻水华暴发中磷的快速补充 机制具有重要的意义. 天然水体中数量巨大的胶体粒子可以通过吸附一解吸过程直接或间接的影响 着物质和元素的生物地球化学循环过程 ${ }^{[17-18]}$. 那么, 对天然水体胶体相可酶解磷含量及分布的研究 将有助于探究水体磷循环过程.

本文针对太湖胥口湾、梅梁湾、望虞河口、千岛湖和富春江 5 个生态系统类型截然不同的水体，进 行了胶体及相关颗粒态物质中可酶解磷(EHP)特征的研究, 以期为进一步认识天然水体磷的循环过程提 供科学依据.

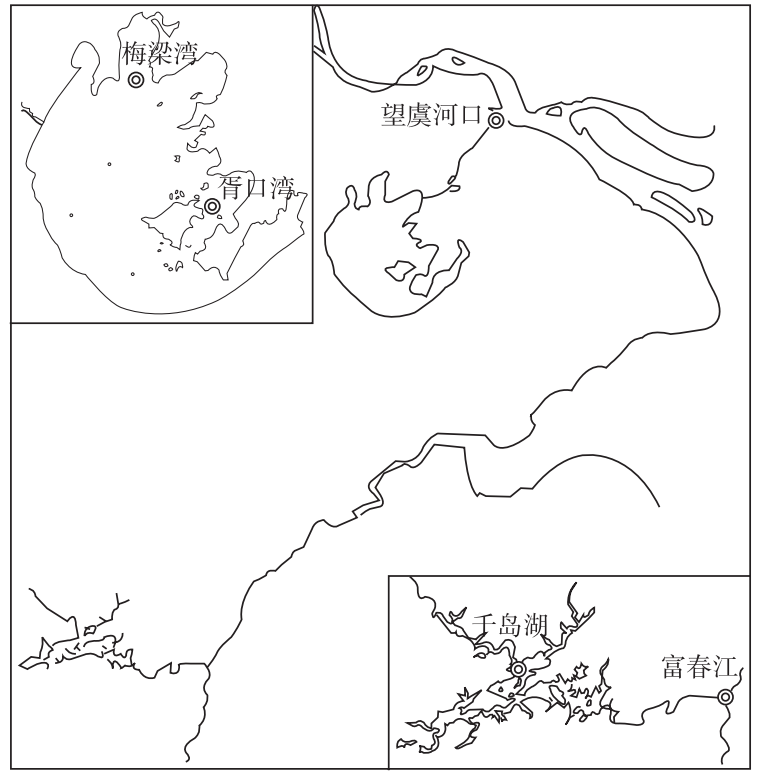

图 1 采样点位置示意图

Fig.1 Location of sampling sites

\section{1 研究区域}

采样点的位置见图 1. 太湖是我国第三大 淡水湖 $\left(30^{\circ} 56^{\prime}-31^{\circ} 56^{\prime} \mathrm{N}, 119^{\circ} 54^{\prime}-120^{\circ} 36^{\prime} \mathrm{E}\right)$, 面 积 $2338 \mathrm{~km}^{2}$, 平均水深 $1.89 \mathrm{~m}$, 最大水深不超过 $3 \mathrm{~m}$, 是一个典型的大型浅水湖泊 ${ }^{[19]}$. 太湖东部 胥口湾水质较好, 沉水植物生长茂盛, 为典型 的浅水草型湖区. 太湖北部的梅梁湾以浮游植 物占优势, 每年 5-10 月都会发生蓝藻水华, 7、 8 月最为严重 ${ }^{[20-21]}$.

望虞河是连接太湖与长江的重要流域性 河流，是“引江济太”工程的骨干河道. 从长江 引水可以补充太湖的水量, 缩短太湖水交换 周期, 增加太湖的水环境容量, 从而缓解太湖 的富营养状况 ${ }^{[22]}$. 本研究选择望虞河口闸外 作为研究水体, 考察其水体中磷的形态和粒 级间分布特征.

千岛湖 $\left(29^{\circ} 22^{\prime}-29^{\circ} 50^{\prime} \mathrm{N}, 118^{\circ} 34^{\prime}-119^{\circ} 15^{\prime} \mathrm{E}\right)$ 位于浙江省西北部, 是 20 世纪 50 年代修建新 安江电站大坝拦水形成的大型人工水库. 与长 江中下游地区的浅水湖泊不同, 千岛湖面积

$573 \mathrm{~km}^{2}$, 平均水深 $34 \mathrm{~m}$, 属大型深水湖泊, 具有与浅水湖泊显著不同的水动力特征. 由于缺乏湖滨水陆 交错带和水生高等植物, 水体初级生产力低, 目前的千岛湖水体水质除湖泊水库特定项目外(全湖总氮年 均为 $0.637 \mathrm{mg} / \mathrm{L}$, 总磷年均为 $0.018 \mathrm{mg} / \mathrm{L}$ ), 主要水质指标均满足地表水的 I 类标准 ${ }^{[23]}$.

千岛湖下游为新安江，在梅城与兰江相汇，以下河段至桐庐称为富春江. 富春江下游建有富春江大 坝, 是一个过流型水库发电站, 不蓄水. 2004 年 7 月, 富春江水库首次发生蓝藻水华, 引起轰动. 本研究 
在梅城三都大桥下游约 $500 \mathrm{~m}$ 处采样, 考察其水体中磷的形态和粒级间分布特征.

\section{2 材料与方法}

\section{1 样品采集}

2007 年 11 月和 12 月, 相继在 5 个不同生态类型的水域采集表层水. 用小型直流葲抽取 $25 \mathrm{~L}$ 表层水 (水下 $25 \mathrm{~cm}$ ), 经 $64 \mu \mathrm{m}$ 篮网过滤后(去除悬浮杂质和较大生物)保存于氟化聚乙烯瓶中, 所有容器依次均经 过 $10 \% \mathrm{HCl}$ 溶液浸泡、去离子水清洗和待收集水样润洗. 水样采回后, 立即进行预过滤. 水样依次通过 $1 \mu \mathrm{m}$ 滤芯和 $0.5 \mu \mathrm{m}$ 滤芯的预过滤系统(10inch Millipore prefilter), 经过 $0.5 \mu \mathrm{m}$ 滤芯的滤液用于切向流超滤. 超滤系统(Millipore Standard Pellicon System)分为两级, 所用超滤膜的孔径分别为 $0.1 \mu \mathrm{m}$ 和 $1 \mathrm{kDa}$ (相当于 $1 \mathrm{~nm})$. 在整个预过滤和超滤过程中分别收集各级滤液和保留液, 得到包括原水样 (滤过 $64 \mu \mathrm{m}$ 篮网)在内的 7 级样品, 待测各化学指标. 为了提高准确性, 每个湖区(河口)均采 3 个平行样.

超滤系统在处理水样之前用去离子水, $0.1 \mathrm{~mol} / \mathrm{L}$ 的 $\mathrm{NaOH}$ 溶液和 $0.1 \mathrm{~mol} / \mathrm{L}$ 的 $\mathrm{HCl}$ 溶液进行严格清洗, 清洗后再用 1-2L 待处理样品循环 10-15 min 对系统进行润洗, 具体方法参考文献[24]. 水样经过超滤处理 后，所得到的保留液是某种粒径胶体粒子被浓缩若干倍数的胶体浓缩液，而透过液是不含该粒径及以上 粒子的超滤液. 水样的浓缩系数可以表示为 $F=V_{\mathrm{p}} / V_{\mathrm{r}}$, 其中 $V_{\mathrm{p}}$ 为预滤液体积, $V_{\mathrm{r}}$ 为保留液体积.

\section{2 分析方法}

2.2 .1 水体可酶解磷的测定 将 $100 \mathrm{ml}$ 各级样品放人具塞、灭菌的三角烧瓶中, 加人 $1 \mathrm{ml} 1.0 \mathrm{~mol} / \mathrm{L}$ Tris 缓 冲溶液 $(\mathrm{pH}=8.4)$ 及 $5 \mathrm{ml}$ 氯仿，在 $30^{\circ} \mathrm{C}$ 条件下处理 4-6d. 通过测定处理前后水体中 $\mathrm{PO}_{4}{ }^{3}$-的浓度差，即可计 算出样品中的可酶解磷 $(\mathrm{EHP})^{[6,25]}$. 为了提高可酶解磷测定的准确性, 待测的每个样品都取 3 个平行样. 本文中总可酶解磷是指滤过 $64 \mu \mathrm{m}$ 的水样中的可酶解磷含量.

2.2.2 磷的测定 各级样品中磷含量的测定采用碱性过硫酸钾消解后钿锑抗分光光度法 ${ }^{[26]}$; 正磷酸根浓度 用锄锑抗分光光度法测定或微量流动注射仪 Skalar(检测限 $0.001 \mathrm{mg} / \mathrm{L}$ ) 测定. 文中的总磷 (TP)是指滤过 $64 \mu \mathrm{m}$ 的水样中的磷含量, 总溶解磷(TDP)则是指水样经 $0.5 \mu \mathrm{m}$ 滤膜过滤后滤液中的磷含量.

根据实验所用滤膜孔径, 本文对各粒级的定义如下: 粒径 1-64 $\mu \mathrm{m}$ 为颗粒相, $1 \mathrm{~nm}-1 \mu \mathrm{m}$ 之间为胶体相, 小于 $1 \mathrm{~nm}$ 为真溶解相. 对于胶体, 作如下细分: $1 \mathrm{~nm}-0.1 \mu \mathrm{m}$ 为微胶体, $0.1-0.5 \mu \mathrm{m}$ 为中胶体, $0.5-1 \mu \mathrm{m}$ 为较 大胶体. 除胶体外, 各粒级的磷含量由其相应上下限的两级样品磷含量相减得到, 如: $1-64 \mu \mathrm{m}$ 颗粒相磷 含量等于透过 $64 \mu \mathrm{m}$ 滤网的滤液 1 与透过 $1 \mu \mathrm{m}$ 滤芯的滤液 2 的磷浓度之差. 胶体相磷含量则由保留液磷 含量等根据公式计算得到: $P_{\mathrm{c}}=\left(P_{\mathrm{r}}-P_{\mathrm{u}}\right) / F$. 式中, $P_{\mathrm{c}}$ 为胶体磷浓度, $P_{\mathrm{r}}$ 为保留液的磷浓度, $P_{\mathrm{u}}$ 为超滤液的磷 浓度, $F$ 为浓缩系数.

\section{3 结果与讨论}

\section{1 可酶解磷含量}

3.1.1 天然水体中的 EHP 含量 表 1 为五种典型水体的营养盐状况和不同粒级的可酶解磷含量. 由于每个 水体采集 3 个平行样, 分别处理得到各级样品, 在测定可酶解磷含量时每个样品再分别取 3 个平行样, 因 此可酶解磷数据的误差来自于采样、过滤超滤、取样及测定各个过程, 误差相对较大. 尽管如此, 本次 调查的 5 种典型水体的可酶解磷含量及分布还是有着显著差别的.

调查结果显示, 太湖胥口湾水体中的总磷 $(0.018 \mathrm{mg} / \mathrm{L})$ 和总可酶解磷 $(0.010 \mathrm{mg} / \mathrm{L})$ 含量均很低, 梅梁湾 水体中的总磷 $(0.121 \mathrm{mg} / \mathrm{L})$ 和总可酶解磷 $(0.026 \mathrm{mg} / \mathrm{L})$ 含量则较高, 两个湖区的总可酶解磷含量存在着明显 差异. 长江-望虞河口总磷 $(0.216 \mathrm{mg} / \mathrm{L})$ 并非最高, 然而其总可酶解磷含量最高, 达 $0.174 \mathrm{mg} / \mathrm{L}$, 远高于其 他 4 个研究水体. 千岛湖水质较好, 而其下游的富春江水库总磷浓度最高 $(0.397 \mathrm{mg} / \mathrm{L})$, 总可酶解磷含量 也比较高 $(0.050 \mathrm{mg} / \mathrm{L})$. 在可酶解磷的分布上, 结果表明颗粒相潜在的可酶解磷含量较高, 胶体相可酶解 磷也占了相当的比重, 而真溶解相的可酶解磷, 5 个研究水体的含量都很低.

此外, 望虞河口和富春江水库水体的 TN、TP 浓度明显高于梅梁湾, 且由两者颗粒相可酶解磷含量高 可知其沉积物中也含有丰富的磷, 具备发生蓝藻水华的营养物质基础. 
3.1.2 藻型湖区水体 EHP 高于草型湖区水体 本次调查结果表明, 胥口湾水体中的总磷 $(0.018 \mathrm{mg} / \mathrm{L})$ 和总 可酶解磷 $(0.010 \mathrm{mg} / \mathrm{L})$ 含量相当低, 而梅梁湾水体中的总磷 $(0.121 \mathrm{mg} / \mathrm{L})$ 和总可酶解磷 $(0.026 \mathrm{mg} / \mathrm{L})$ 含量比 较高, 两个湖区的总可酶解磷含量存在着明显差异, 这与各自的生态系统特征是相对应的.

表 15 种水体营养盐状况及不同粒级的可酶解磷含量 $(\mathrm{mg} / \mathrm{L})$

Tab.1 EHP concentration in different fractions of water samples

\begin{tabular}{cccccc}
\hline & 胥口湾 & 梅梁湾 & 望虞河口 & 千岛湖 & 富春江 \\
\hline 生态系统类型 & 草型湖区 & 藻型湖区 & 河流人长江口 & 大型深水水库 & 河流型水库 \\
$\mathrm{TN}(<64 \mu \mathrm{m})$ & $0.545 \pm 0.040$ & $1.638 \pm 0.033$ & $2.472 \pm 0.052$ & $1.094 \pm 0.068$ & $3.416 \pm 0.086$ \\
$\mathrm{TP}(<64 \mu \mathrm{m})$ & $0.018 \pm 0.001$ & $0.121 \pm 0.014$ & $0.216 \pm 0.001$ & $0.045 \pm 0.009$ & $0.397 \pm 0.031$ \\
颗粒相 $(1-64 \mu \mathrm{m}) \mathrm{EHP}$ & $0.005 \pm 0.003$ & $0.014 \pm 0.009$ & $0.131 \pm 0.040$ & $0.002 \pm 0.001$ & $0.022 \pm 0.025$ \\
较大胶体 $(0.5-1 \mu \mathrm{m}) \mathrm{EHP}$ & $0.001 \pm 0.002$ & $0.001 \pm 0.001$ & $0.029 \pm 0.013$ & $0.000 \pm 0.002$ & $0.007 \pm 0.006$ \\
中胶体 $(0.1-0.5 \mu \mathrm{m}) \mathrm{EHP}$ & $0.002 \pm 0.000$ & $0.002 \pm 0.001$ & $0.005 \pm 0.003$ & $0.001 \pm 0.000$ & $0.006 \pm 0.003$ \\
微胶体 $(1 \mathrm{~nm}-0.1 \mu \mathrm{m}) \mathrm{EHP}$ & $0.000 \pm 0.000$ & $0.002 \pm 0.000$ & $0.003 \pm 0.000$ & $0.001 \pm 0.000$ & $0.008 \pm 0.003$ \\
真溶解相 $(<1 \mathrm{~nm}) \mathrm{EHP}$ & $0.000 \pm 0.002$ & $0.005 \pm 0.001$ & $0.001 \pm 0.002$ & $0.001 \pm 0.001$ & $0.002 \pm 0.008$ \\
胶体相 $(1 \mathrm{~nm}-1 \mu \mathrm{m}) \mathrm{EHP}$ & $0.003 \pm 0.002$ & $0.004 \pm 0.001$ & $0.037 \pm 0.014$ & $0.002 \pm 0.002$ & $0.020 \pm 0.007$ \\
总可酶解磷 $(1 \mathrm{~nm}-1 \mu \mathrm{m})$ & $0.010 \pm 0.002$ & $0.026 \pm 0.009$ & $0.174 \pm 0.039$ & $0.003 \pm 0.001$ & $0.050 \pm 0.025$ \\
\hline
\end{tabular}

太湖东部的胥口湾水质较好, 沉水植物生长茂盛, 为典型的浅水草型湖区, 其沉积物中的磷组分以 相对较难被生物利用的有机磷为主 ${ }^{[27-28]}$. 沉积物的组成特征及沉水植物对底泥的固定作用使得胥口湾沉 积物中的磷释放很小, 又由于沉水植物生长对水体中磷的大量吸收, 胥口湾水体的磷含量非常低. 而梅 梁湾属富营养化湖区，每年 5-10 月都会暴发蓝藻水华 ${ }^{[20-21]}$. 其沉积物中磷组分以无机磷为主，易被生物 利用的有效磷含量较高 ${ }^{[29]}$, 水面复氧较草型湖区强烈 ${ }^{[30]}$, 风浪导致的再悬浮作用也比草型湖区强烈, 释 放出来的磷进人上覆水后更容易被氧化和被颗粒物吸附. 此外，藻类不断的死亡、分解行为，产生大量的 颗粒物质和胶体物质悬浮在水体中. 因此, 梅梁湾水体的磷含量和可酶解磷含量均显著高于胥口湾水体.

目前普遍认为, 当水体中磷酸根含量不足以维持藻类的生长、繁殖及其他水体相关代谢时, 生物体 能够通过碱性磷酸酶等物质的催化作用, 促进非生物直接利用态的磷转化为生物利用态的磷 ${ }^{[6,10-11]}$. 即 可酶解磷在水体中 DRP 较多时为磷的一个临时储库, 当 DRP 不足时即被生物分泌的或者溶解态的磷酸 酶分解转化为生物利用态的磷. 梅梁湾水体可酶解磷含量远高于胥口湾, 这为梅梁湾的蓝藻水华暴发提 供了更有利的营养条件.

3.1 .3 望虞河水 EHP 高, “引江济太”有风险 调查结果显示, 长江-望虞河口总磷 $0.216 \mathrm{mg} / \mathrm{L}$, 而其总可酶 解磷含量高达 $0.174 \mathrm{mg} / \mathrm{L}$, 远高于其他四个研究水体. 目前, 望虞河并未发生水华, 一方面是因为蓝 藻水华的发生除了要满足营养盐条件外, 还受水体细菌群落结构、水下光场特征等影响. 以太湖为例, 太湖蓝藻水华最严重的区域发生在梅梁湾、竺山湾等北部湖湾地区，但是营养盐更高、污染更严重 的五里湖、北部几个河口地区的水华反而不多. 其原因是这些地区高有机质含量、高有色溶解性有 机物及特殊的细菌群落环境已经不利于微囊藻的生存竞争. 另一方面, 望虞河没有发生蓝藻水华, 还与其水动力条件是分不开的：一般处于封闭或半封闭状态的水体更新量小、自净能力弱，更易生成 蓝藻，而河流中水流较快、水体更新量大，不利于藻类的生长和团聚，不易发生水华. 望虞河口水体 中 TN、TP 及可酶解磷含量远高于太湖梅梁湾蓝藻水华区, 虽然目前望虞河水体没有发生水华, 仍不 排除“引江济太”调水具有一定的生态风险。也就是说在有些情况下，调人太湖的水体中生物可利用 磷的含量很高, 会增加太湖水体中生物可利用磷的含量. 调水进人太湖后, 一旦水动力条件和光场 条件等适宜, 同样有可能引发大规模的水华. 2004 年的富春江水华事件就是水动力条件发生改变导致 蓝藻暴发的例子. 
本调查显示富春江水库的 TP 最高 $(0.397 \mathrm{mg} / \mathrm{L})$, EHP 含量也比较高 $(0.050 \mathrm{mg} / \mathrm{L})$. 与梅梁湾相比，富春 江水库的营养盐浓度完全具备发生蓝藻水华的营养物质基础, 水华的发生主要取决于水文和气象条件. 2004 年 7 月，富春江及钱塘江闻家堰至钱塘江大桥河段发生了大面积蓝藻爆发，当时发生水华的关键条 件就是夏季降雨仅为正常年份的三分之一，特别低的来水补充使得富春江水库的换水周期大大延长，而 水力停留时间的大大增加使得蓝藻生物量在水体大量积累并形成水华.

因此, TP 和 EHP 含量很高的望虞河河水调人太湖后, 虽然可以补充太湖的水量, 缩短太湖水交换周 期, 但也可能造成太湖水体中生物有效磷浓度的增加. 如果水文和气象条件等适合, 仍有可能暴发大规 模的水华. 因此, 在选择调水时机上应该特别注意加强对引调水源的水质监测, 为科学调水的具体实施 和预测提供依据.

\section{2 可酶解磷的分布}

3.2.1 颗粒相和胶体相可酶解磷是生物必需磷的重要储库 有研究表明, 海域中尤其是河口和近岸海域颗 粒态潜在的生物可利用性磷占总磷比例较高, 是生物必需磷的一个不可忽视的来源和重要储库, 也是控 制海域磷供应水平的重要因子 ${ }^{[12-16]}$. 通过对太湖胥口湾、梅梁湾、望虞河口、千岛湖和富春江五种典型 水体可酶解磷含量的研究发现, 颗粒态潜在的可酶解磷含量占总可酶解磷含量的比重较高(图 2), 分别达 到 $46.3 \% 、 53.6 \% 、 74.9 \% 、 46.1 \% 、 44.4 \%$ ，与海洋、河口的研究结果一致. 同时, 胶体态的可酶解磷也占 了相当的比重(图 2), 分别达到 $31.8 \% 、 16.6 \% 、 21.2 \% 、 55.6 \% 、 40.7 \%$, 是生物必需磷不可忽视的重要储 库. 真溶解相的可酶解磷, 5 个研究水体的含量都很低, 只有梅梁湾略高(表 1); 而其占总可酶解磷的比重, 则是梅梁湾和千岛湖所占的比例稍高(图 2).

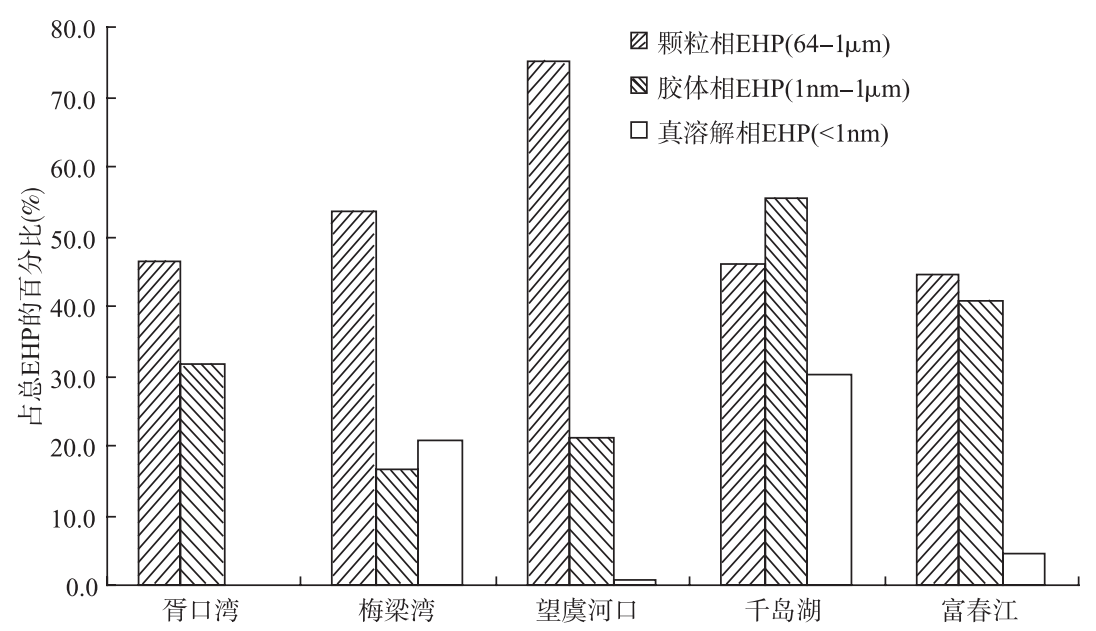

图 2 颗粒、胶体、真溶解相可酶解磷含量占总可酶解磷的百分比

Fig.2 Percentage concentration of EHP in different fractions of water samples

3.2.2 胶体相可酶解磷的分布特征 作为颗粒相和真溶解相之间的联系, 胶体颗粒非常微小, 在天然水体 中可以比颗粒物更加稳定的存在, 具有独特的迁移能力和活跃的化学性质 ${ }^{[31-33]}$. 数量巨大的胶体粒子可 以通过吸附一解吸过程影响着物质和元素的生物地球化学循环过程 ${ }^{[17-18]}$, 且部分胶体颗粒本身就含有磷, 因此胶体及胶体相可酶解磷在天然水体的磷循环中应该起着重要的作用.

从图 3 可以看到三种趋势: 胥口湾、望虞河的胶体态可酶解磷主要分布在较大、中胶体范围, 真溶 解相的可酶解磷含量很少; 梅梁湾和千岛湖的胶体态可酶解磷主要分布在中、微胶体范围, 同时真溶解 相也含有很大比重的可酶解磷; 富春江水库的胶体态可酶解磷分布的相对比较均匀, 真溶解相的可酶解 磷所占的比重也在前两类之间，属于中间状态. 


\section{4 结论}

本文通过对太湖胥口湾、梅梁湾、望虞河口、千岛湖和富春江五种典型水体的可酶解磷含量及分布 特征的研究, 得出以下结论:

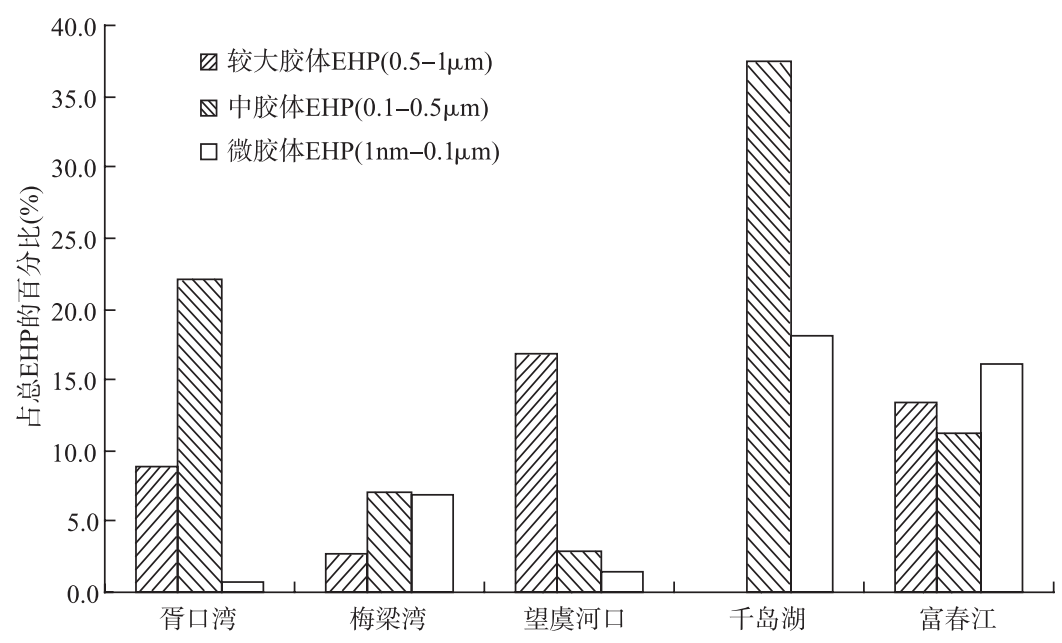

图 3 不同粒级胶体的可酶解磷含量占总可酶解磷的百分比

Fig.3 Percentage concentration of EHP in different fractions of colloid samples

(1) 5 种典型水体中颗粒态潜在的可酶解磷含量占总可酶解磷含量的比重较高, 分别达到 $46.3 \%$ 、 $53.6 \% 、 74.9 \% 、 46.1 \% 、 44.4 \%$, 与海洋、河口的研究结果一致; 胶体态的可酶解磷也占了相当的比重, 分 别达到 $31.8 \% 、 16.6 \% 、 21.2 \% 、 55.6 \% 、 40.7 \%$ ，是生物必需磷不可忽视的重要储库.

(2) 望虞河口河水的磷和可酶解磷含量较高, 其输人可能对太湖水体生物有效磷浓度的增加起到重 要的贡献, “引江济太”调水时具有一定生态风险，应注意调水时机.

(3) 对于胶体范围 $(1 \mathrm{~nm}-1 \mu \mathrm{m})$ 的可酶解磷, 胥口湾、望虞河的胶体态可酶解磷主要分布在较大、中胶 体范围; 梅梁湾和千岛湖的胶体态可酶解磷主要分布在中、微胶体范围; 富春江水库的胶体态可酶解磷 分布的相对比较均匀.

致谢: 高光研究员在研究中给予指导与帮助，薛静琛同志在实验分析上给予很大帮助，在此表示衰 心感谢.

\section{5 参考文献}

[1] Qin BQ, Zhu GW. The nutrient forms, cycling and exchange flux in the sediment and overlying water system in lakes from the middle and lower reaches of Yangtze River. Sci China Ser D-Earth Sci, 2006, 49(S1): 1-13.

[2] Schindler DW. Evolution of phosphorus limitation in lakes. Science, 1977, 195: 260-262.

[3] Smith VH. Low nitrogen to phosphorus ratios favor dominance by blue-green algae in lake phytoplankton. Science, 1983, 221: 669-671.

[4] Fan CX, Zhang L, Qin BQ et al. Estimation on dynamic release of phosphorus from wind-induced suspended particulate matter in Lake Taihu. Sci China Ser D-Earth Sci, 2004, 47(8): 710-719.

[5] 朱广伟, 秦伯强, 张 路. 长江中下游湖泊沉积物中磷的形态及藻类可利用量. 中国科学( D 辑): 地球科学, 2005, 35(增 刊 II ): 24-32.

[6] 高 光, 朱广伟, 秦伯强等. 太湖水体中碱性磷酸酶的活性及磷的矿化速率. 中国科学(D 辑): 地球科学, 2005, 35(增刊 II ): $157-165$. 
[7] 谢 平. 浅水湖泊内源磷负荷季节变化的生物驱动机制. 中国科学(D 辑): 地球科学, 2005, 35(增刊 II ): 11-23.

[8] 张 敏, 谢 平, 徐 军等. 大型浅水湖泊——巢湖内源磷负荷的时空变化特征及形成机制. 中国科学(D辑): 地球科学, 2005, 35(增刊 II): 63-72.

[9] Halemejko GZ, Chróst RJ. Role of phosphatases in phosphorus mineralization during decomposition of lake phytoplankton blooms. Archiv für Hydrobiologie, 1984, 101(4): 489-502.

[10] Song CL, Cao XY, Zhou YY et al. Contributions of phosphatase and microbial activity to internal phosphorus loading and their relation to lake eutrophication. Sci China Ser D-Earth Sci, 2006, 49(S1): 102-111.

[11] Zhou YY, Li JQ, Song CL et al. Variations and possible source of potentially available phosphorus in a Chineses shallow eutrophic lake. Journal of Freshwater Ecology, 2004, 19(1): 87-96.

[12] 郑爱榕, 沈海维, 李文权. 沉积物中磷的存在形态及其生物可利用性研究. 海洋学报, 2004, 26(4): 49-57.

[13] Perkins RG, Underwood GJC. The potential for phosphorus release across the sediment -water interface in an eutrophic reservoir dosed with ferric sulphate. Water Research, 2001, 35(6): 1399-1406.

[14] Person G. Phosphorus in tributaries to Lake Malaren, Sweden: analytical fractions, anthropogenic contribution and bioavailability. Ambio, 2001, 30(8): 486-495.

[15] Zhou QX, Christopher EG, Zhu YM. Evaluation of phosphorus bioavailability in sediments of there contrasting lakes in China and the UK. Chemosphere, 2001, 42: 221-225.

[16] Jensen HS, Mcglathery KJ, Marino et al. Forms and availability of sediment phosphorus in carbonate sand of Bermuda seagrass beds. Limnology and Oceanography, 1998, 43(5): 799-810

[17] Honeyman BD, Santschi PH. A Brownian-pumping model for oceanic trace metal scavenging: Evidence from Th isotopes. $J$ Mar Res, 1989, 47: 951-992.

[18] Honeyman BD, Santschi PH. Coupling adsorption and particles aggregation: Laboratory studies of "Colloidal Pumping" using ${ }^{59} \mathrm{Fe}$-labeled hematite. Environmental Science \& Technology, 1991, 25: 1739-1747.

[19] 秦伯强, 胡维平, 陈伟民. 太湖水环境演化的过程与机理. 北京: 科学出版社, 2004: 1-40.

[20] Chen YW, Fan CX, Teubner K et al. Changes of nutrients and phytoplankton chlorophyll-a in a large shallow lake, Taihu, China: an 8-year investigation. Hydrobiologia, 2003, 506/509: 273-279.

[21] Chen YW, Qin BQ, Teubner K et al. Long-term dynamics of phytoplankton assemblages: Microcystis-domination in Lake Taihu, a large shallow lake in China. Journal of Plankton Research, 2003, 25(1): 445-453.

[22] 吴挺峰, 潘彩英, 崔广柏等. 引江济太水源地水质及河势可靠性分析. 河海大学学报(自然科学版), 2007, 35(4): 369-373.

[23] 赵新民, 王 军, 朱淑君. 千岛湖水体叶绿素 a 时空变化特征及其影响因子分析. 浙江树人大学学报, 2005, 5(5): 99-103.

[24] 张战平, 孙小静, 楼章华等. 太湖春季水体中的胶体有机碳含量及影响因素分析. 中国环境科学, 2006, 26(2): 166-170.

[25] Chróst RJ, Siuda W, Albrecht D et al. A method for determining enzymatically hydrolysable phosphorus (EHP) in nature waters. Limnology and Oceanography, 1986, 31: 662-667.

[26] 国家环境保护总局《水和废水监测分析方法》编委会. 水和废水监测分析方法(第 4 版). 北京: 中国环境科学出版社, 2002: $246-248$

[27] 朱广伟, 高 光, 秦伯强等. 浅水湖泊沉积物中磷的地球化学特征. 水科学进展, 2003, 14(6): 714-719.

[28] 朱广伟, 秦伯强, 高 光等. 长江中下游浅水湖泊沉积物中磷的形态及其与水相磷的关系. 环境科学学报, 2004, 24(3): 381-388.

[29] 黄清辉, 王东红, 王春霞等. 沉积物中磷形态与湖泊富营养化的关系. 中国环境科学, 2003, 23(6): 583-586

[30] 张 路, 范成新, 王建军等. 太湖草藻型湖区间隙水理化特性比较. 中国环境科学, 2004, 24(5): 556-560.

[31] Gustafson O, Gschwend PM. Acuatic colloids: concepts, definitions and current challenges. Limnology and Oceanography, 1997, 42: 519-528.

[32] Dai MH, Benitez-Nelson CR. Colloidal organic carbon and $\mathrm{Th}^{234}$ in the Gulf of Maine. Marine Chemistry, 2001, 74: 181-196.

[33] Wells ML, Goldberg ED. Occurrence of small colloids in sea water. Nature, 1991, 353: 342-344. 